\title{
On the mean square of Lerch zeta-functions
}

\author{
R. Garunkštis*, A. Laurinčikas ${ }^{\dagger}$ J. Steuding ${ }^{\ddagger}$
}

March 2001

\begin{abstract}
Using the approximate functional equation for Lerch zeta-functions $L(\lambda, \alpha, s)=\sum_{n=0}^{\infty} \frac{e(\lambda n)}{(n+\alpha)^{s}}$, we prove for fixed parameters $0<\lambda, \alpha \leq 1$ asymptotic formulas for the mean square of $L(\lambda, \alpha, s)$ inside the critical strip. This improves earlier results of D. Klusch and of A. Laurinčikas.
\end{abstract}

Keywords: Lerch zeta-function, mean square.

AMS subject classification: 11M35.

\section{Introduction and statement of results}

As usual let $s=\sigma+i t$ and $e(z)=\exp (2 \pi i z)$. Further, denote by $[\lambda]$ the integral and by $\{\lambda\}$ the fractional part of a real number $\lambda$. For the parameters $0<\alpha \leq 1, \lambda \in \mathbb{R}$ the Lerch zeta-function is given by

$$
L(\lambda, \alpha, s)=\sum_{n=0}^{\infty} \frac{e(\lambda n)}{(n+\alpha)^{s}} \quad(\sigma>1) .
$$

*Department of Mathematics and Informatics, Vilnius University, Naugarduko 24, 2600 Vilnius, Lithuania, e-mail: ramunas.garunkstis@maf.vu.lt, home page: www.mif.vu.lt/ttsk/bylos/ga/ga_a.html Partially supported by Grant from Lithuanian Foundation of Studies and Science.

${ }^{\dagger}$ Department of Mathematics and Informatics, Vilnius University, Naugarduko 24, 2600 Vilnius, Lithuania, e-mail: antanas.laurincikas@maf.vu.lt

Partially supported by Grant from Lithuanian Foundation of Studies and Science.

${ }_{\ddagger}^{\ddagger}$ Mathematisches Seminar, Johann Wolfgang Goethe-Universität Frankfurt, Robert-Mayer-Str. 10, D-60054 Frankfurt, Germany, e-mail: steuding@math.uni-frankfurt.de

The third author would like to express his deep gratitude to the kind hospitality at the Department of Number theory and Probability theory at Vilnius University. 
Obviously $L(\lambda, \alpha, s)=L(\{\lambda\}, \alpha, s)$, so in the sequel we may assume $0<\lambda \leq 1$. Lerch zeta-functions have an analytical continuation to the whole complex plane except for at most one simple pole at $s=1 . L(\lambda, \alpha, s)$ is irregular at $s=1$ if and only if $\lambda \in \mathbb{Z}$, in which case the Lerch zeta-function becomes the so-called Hurwitz zeta-function $\zeta(s, \alpha):=$ $L(1, \alpha, s)$; especially for $\lambda=\alpha=1$ we obtain the well known Riemann zeta-function. In any case, Lerch zeta-functions satisfy the functional equation

$$
\begin{aligned}
L(\lambda, \alpha, 1-s)= & \frac{\Gamma(s)}{(2 \pi)^{s}}\left(e\left(\frac{s}{4}-\alpha \lambda\right) L(-\alpha, \lambda, s)\right. \\
& \left.+e\left(-\frac{s}{4}+\alpha(1-\{\lambda\})\right) L(\alpha, 1-\{\lambda\}, s)\right),
\end{aligned}
$$

which was first proved by Lerch [10] for $0<\lambda<1$, and by Hurwitz [6] for $\lambda=1$.

For analytical investigations it is often useful to deal with approximations. For example, if $0 \leq \sigma_{0} \leq \sigma \leq 2,0<|t| \leq \pi \lambda N$, then

$$
L(\lambda, \alpha, s)=\sum_{0 \leq n \leq N} \frac{e(\lambda m)}{(n+\alpha)^{s}}+[\lambda] \frac{N^{1-s}}{s-1}+O\left(N^{-\sigma}\right) .
$$

A proof of (2) for $\lambda=1$ and $\alpha=1$, the case of the Riemann zeta-function, can be found in [7]; for $\lambda=1,0<\alpha \leq 1$, the case of Hurwitz zeta-function, follows with the same arguments; for Lerch zeta-function this formula was proved in [3]. In [4] the authors proved the following approximate functional equation (which combines the functional equation (1) and approximating Dirichlet polynomials like the one in (2)): if $0<\lambda, \alpha \leq 1$ and $0 \leq \sigma \leq 1$ are fixed, then we have, for $t \geq 1$,

$$
\begin{aligned}
L(\lambda, \alpha, s)= & \sum_{0 \leq n \leq m(t)} \frac{e(\lambda n)}{(n+\alpha)^{s}}+\left(\frac{t}{2 \pi}\right)^{\frac{1}{2}-\sigma-i t} e^{i t+\frac{\pi i}{4}-2 \pi i\{\lambda\} \alpha} \sum_{0 \leq n \leq q(t)} \frac{e(-\alpha n)}{(n+\lambda)^{1-s}} \\
& +\left(\frac{t}{2 \pi}\right)^{-\frac{\sigma}{2}} e^{\pi i f(\lambda, \alpha, t)} \psi(g(\lambda, \alpha, t))+O\left(t^{\frac{\sigma}{2}-1}\right)
\end{aligned}
$$

where

$$
\begin{aligned}
m(t):= & {\left[\sqrt{\frac{t}{2 \pi}}-\alpha\right], \quad q(t):=\left[\sqrt{\frac{t}{2 \pi}}\right] } \\
g(\lambda, \alpha, t):= & 2 \sqrt{\frac{t}{2 \pi}}-q(t)-m(t)-\{\lambda\}-\alpha \\
f(\lambda, \alpha, t):= & -\frac{t}{2 \pi} \log \frac{t}{2 \pi e}--\frac{7}{8}+\frac{1}{2}\left(\alpha^{2}-\{\lambda\}^{2}\right)-\alpha(q(t)-m(t)) \\
& +2 \sqrt{\frac{t}{2 \pi}}(q(t)-m(t)+\{\lambda\}-\alpha)--\frac{1}{2}(q(t)+m(t)) \\
& -\{\lambda\}(\alpha+(q(t)-m(t))
\end{aligned}
$$


and

$$
\psi(z):=\frac{\cos \pi\left(\frac{1}{2} z^{2}-z-\frac{1}{8}\right)}{\cos \pi z} .
$$

Note that $\psi(z)$ is everywhere regular. Lets define the constant $c(\alpha)$ by

$$
\sum_{n=0}^{M}(n+\alpha)^{-1}=\log M+c(\alpha)+O(1 / M)
$$

as $M \rightarrow \infty$. Using the approximate functional equation we will prove

Theorem 1 For $0<\lambda, \alpha \leq 1$ we have, as $T$ turns to infinity,

$$
\int_{1}^{T}|L(\lambda, \alpha, 1 / 2+i t)|^{2} d t=T \log \frac{T}{2 \pi}+T(c(\alpha)+c(\lambda)-1)+O\left(T^{\frac{1}{2}} \log T\right)
$$

and for $\frac{1}{2}<\sigma<1$,

$$
\begin{aligned}
\int_{1}^{T}|L(\lambda, \alpha, \sigma+i t)|^{2} d t= & \zeta(2 \sigma, \alpha) T+\frac{(2 \pi)^{2 \sigma-1}}{2-2 \sigma} \zeta(2-2 \sigma, \lambda) T^{2-2 \sigma} \\
& +O\left(T^{1-\sigma} \log T+T^{\frac{\sigma}{2}}\right) .
\end{aligned}
$$

These formulas extend a result of Rane [12] (who proved a mean square formula for Hurwitz zeta-functions on $\sigma=\frac{1}{2}$ with the same error term), improves some results of Klusch [8] (mean square formulas for Lerch zeta-functions for $\frac{1}{2} \leq \sigma<1$ without error term), also improves results from [9], where Theorem 1 by help of the approximation (2) was obtained with the error term $O\left(T^{2-2 \sigma}\right)$.

\section{Preliminaries}

In the proof of Theorem 1 we will often make use of

Lemma 2 For $0<\lambda, \alpha \leq 1$ and $\beta<1, \beta+\gamma<1$ we have

$$
\sum_{0 \leq m<n \leq Q}(m+\alpha)^{-\beta}(n+\lambda)^{-\gamma}\left(\log \frac{n+\lambda}{m+\alpha}\right)^{-1} \ll Q^{2-\beta-\gamma} \log Q .
$$

Proof. We split the sum according to the cases $m<\frac{n}{2}$ and $\frac{n}{2} \leq m<n$. Obviously, we have in the first case

$$
\begin{aligned}
& \sum_{1 \leq n \leq Q} \sum_{m<\frac{n}{2}}(m+\alpha)^{-\beta}(n+\lambda)^{-\gamma}\left(\log \frac{n+\lambda}{m+\alpha}\right)^{-1} \\
& \ll \sum_{1 \leq n \leq Q} n^{-\gamma} \sum_{m<n} m^{-\beta} \ll \sum_{1 \leq n \leq Q} n^{1-\beta-\gamma} \ll Q^{2-\beta-\gamma} .
\end{aligned}
$$


If we write $m=n-r$ in the second case, then we get by the Taylor expansion of the $\operatorname{logarithm} \log \frac{n+\alpha}{m+\lambda} \gg \frac{r}{n}$ for $1 \leq r<\frac{n}{2}$. Thus, we obtain

$$
\begin{gathered}
\sum_{1 \leq n \leq Q} \sum_{\frac{n}{2} \leq m<n}(m+\alpha)^{-\beta}(n+\lambda)^{-\gamma}\left(\log \frac{n+\lambda}{m+\alpha}\right)^{-1} \\
\ll \sum_{1 \leq n \leq Q}(n+\lambda)^{-\gamma} \sum_{1 \leq r<\frac{n}{2}}(n+\alpha-r)^{-\beta} \frac{n}{r} \\
\ll \sum_{1 \leq n \leq Q} n^{1-\beta-\gamma} \sum_{1 \leq r \leq n} \frac{1}{r} \ll Q^{2-\beta-\gamma} \log Q .
\end{gathered}
$$

Collecting both cases together, the estimate of the lemma follows.

Moreover, we need some estimates of exponential integrals.

Lemma 3 (First derivative test) Let $g(t)$ be real and differentiable with monotonic $g^{\prime}(t) \geq \delta_{1}>0$ and $G(t)$ monotonic with $|G(t)| \leq G$ on $[A, B]$. Then

$$
\int_{A}^{B} G(t) e^{i g(t)} d t \ll G \delta_{1}^{-1}
$$

Lemma 4 (Second derivative test) Let $g(t)$ be real, twice differentiable with $g^{\prime \prime}(t) \geq$ $\delta_{2}>0$ throughout $[A, B]$. Then

$$
\int_{A}^{B} e^{i g(t)} d t \ll \delta_{2}^{-\frac{1}{2}}
$$

Proofs can be found in [7]. Note that if $G(t)$ and $g(t)$ both are twice differentiable on $[A, B]$, then one can get for the integral in Lemma 3 by partial integration

$$
\int_{A}^{B} G(t) e^{i g(t)} d t \ll \max _{A \leq t \leq B}\left|\frac{G(t)}{g^{\prime}(t)}\right|+(B-A) \max _{A \leq t \leq B}\left|\frac{G^{\prime}(t) g^{\prime}(t)-G(t) g^{\prime \prime}(t)}{g^{\prime}(t)^{2}}\right| .
$$

\section{Proof of Theorem 1}

Using the approximate functional equation (3), we have

$$
\begin{aligned}
\int_{\frac{T}{2}}^{T} & |L(\lambda, \alpha, \sigma+i t)|^{2} d t \\
= & \int_{\frac{T}{2}}^{T} \sum_{0 \leq m, n \leq m(t)} \frac{e(\lambda(m-n))}{(m+\alpha)^{\sigma+i t}(n+\alpha)^{\sigma-i t}} d t \\
& \quad+\int_{\frac{T}{2}}^{T}\left(\frac{t}{2 \pi}\right)^{1-2 \sigma} \sum_{0 \leq m, n \leq q(t)} \frac{e(\alpha(m-n))}{(m+\lambda)^{1-\sigma-i t}(n+\lambda)^{1-\sigma+i t}} d t
\end{aligned}
$$




$$
\begin{aligned}
& +\int_{\frac{T}{2}}^{T}\left(\frac{t}{2 \pi}\right)^{-\sigma} \psi^{2}(g(\lambda, \alpha, t)) d t+O\left(\int_{\frac{T}{2}}^{T} t^{\sigma-2} d t\right) \\
& +2 \operatorname{Re}\left(\int_{\frac{T}{2}}^{T}\left(\frac{t}{2 \pi}\right)^{\frac{1}{2}-\sigma+i t} e^{-i t-\frac{\pi i}{4}+2 \pi i\{\lambda\} \alpha} \times\right. \\
& \times \sum_{0 \leq m, n \leq q(t)} \frac{e(\lambda m+\alpha n)}{(m+\alpha)^{\sigma+i t}(n+\lambda)^{1-\sigma+i t}} d t \\
& +\int_{\frac{T}{2}}^{T}\left(\frac{t}{2 \pi}\right)^{-\frac{\sigma}{2}} e^{-\pi i f(\lambda, \alpha, s)} \psi(g(\lambda, \alpha, t)) \sum_{0 \leq n \leq m(t)} \frac{e(\lambda n)}{(n+\alpha)^{\sigma+i t}} d t \\
& +\int_{\frac{T}{2}}^{T}\left(\frac{t}{2 \pi}\right)^{\frac{1}{2}-\frac{3 \sigma}{2}-i t} e^{i t+\frac{\pi i}{4}-2 \pi i\{\lambda\} \alpha-\pi i f(\lambda, \alpha, s)} \psi(g(\lambda, \alpha, t)) \times \\
& \times \sum_{0 \leq n \leq q(t)} \frac{e(-\alpha n)}{(n+\lambda)^{1-\sigma-i t}} d t+\int_{\frac{T}{2}}^{T} O\left(t^{\frac{\sigma}{2}-1}\right) \sum_{0 \leq n \leq m(t)} \frac{e(\lambda n)}{(n+\alpha)^{\sigma+i t}} d t \\
& +\int_{\frac{T}{2}}^{T} O\left(t^{\frac{\sigma}{2}-1}\right)\left(\frac{t}{2 \pi}\right)^{\frac{1}{2}-\sigma-i t} e^{i t+\frac{\pi i}{4}-2 \pi i\{\lambda\} \alpha} \sum_{0 \leq n \leq q(t)} \frac{e(-\alpha n)}{(n+\lambda)^{1-\sigma-i t}} d t \\
& +\int_{\frac{T}{2}}^{T} O\left(t^{\frac{\sigma}{2}-1}\right)\left(\frac{t}{2 \pi}\right)^{-\frac{\sigma}{2}} e^{\pi i f(\lambda, \alpha, s)} \psi(g(\lambda, \alpha, t) d t) \\
& \quad \sum_{1 \leq j \leq 10} \mathcal{I}_{j} .
\end{aligned}
$$

First, we find

$$
\mathcal{I}_{1}=\sum_{0 \leq m, n \leq m(T)} \frac{e(\lambda(n-m))}{(m+\alpha)^{\sigma}(n+\alpha)^{\sigma}} \int_{T_{1}}^{T}\left(\frac{m+\alpha}{n+\alpha}\right)^{i t} d t
$$

with $T_{1}:=\max \left\{\frac{T}{2}, 2 \pi(m+\alpha)^{2}, 2 \pi(n+\alpha)^{2}\right\}$. The contribution of the diagonal terms is

$$
\begin{aligned}
& \sum_{0 \leq n \leq m(T)}(n+\alpha)^{-2 \sigma}\left(T-\max \left\{\frac{T}{2}, 2 \pi(n+\alpha)^{2}\right\}\right) \\
& =T \sum_{0 \leq n \leq m(T)}(n+\alpha)^{-2 \sigma}-\frac{T}{2} \sum_{0 \leq n \leq m\left(\frac{T}{2}\right)}(n+\alpha)^{-2 \sigma} \\
& -2 \pi \sum_{m\left(\frac{T}{2}\right)+1 \leq n \leq m(T)}(n+\alpha)^{2-2 \sigma} .
\end{aligned}
$$

for $\sigma=1 / 2$ this is equal to

$$
\frac{T}{4} \log \frac{T}{\pi}+\frac{T}{2}\left(c(\alpha)-\frac{1}{2}\right)+O\left(T^{1 / 2}\right) .
$$


For $1 / 2<\sigma<1$, by use of (2) the diagonal terms above equal

$$
\frac{1}{2} \zeta(2 \sigma, \alpha) T-\frac{2(2 \pi)^{\sigma-\frac{1}{2}}\left(1-2^{\sigma-\frac{3}{2}}\right)}{(2 \sigma-1)(3-2 \sigma)} T^{\frac{3}{2}-\sigma}+O\left(T^{1-\sigma}\right) .
$$

The non-diagonal terms are

$$
\begin{aligned}
& \sum_{\substack{0 \leq m, n \leq m(T) \\
m \neq n}} \frac{e(\lambda(n-m))}{(m+\alpha)^{\sigma}(n+\alpha)^{\sigma}} \int_{T_{1}}^{T} \exp \left(i t \log \frac{m+\alpha}{n+\alpha}\right) d t \\
= & \sum_{\substack{0 \leq m, n \leq m(T) \\
m \neq n}} \frac{e(\lambda(n-m))}{(m+\alpha)^{\sigma}(n+\alpha)^{\sigma}}\left(i \log \frac{m+\alpha}{n+\alpha}\right)^{-1} \times \\
\times & \sum_{0 \leq m<n \leq m(T)}\left(\exp \left(i T \log \frac{m+\alpha}{n+\alpha}\right)--\exp \left(i T_{1} \log \frac{m+\alpha}{n+\alpha}\right)\right) \\
\ll & \sum_{0 \leq m}^{-\sigma}(n+\alpha)^{-\sigma}\left(\log \frac{n+\alpha}{m+\alpha}\right)^{-1} .
\end{aligned}
$$

Applying Lemma 2, the non-diagonal terms turn out to be $\ll T^{1-\sigma} \log T$. Hence, for $\sigma=1 / 2$,

$$
\mathcal{I}_{1}=\frac{T}{4} \log \frac{T}{\pi}+\frac{T}{2}\left(c(\alpha)-\frac{1}{2}\right)+O\left(T^{1 / 2} \log T\right),
$$

and, for $1 / 2<\sigma<1$,

$$
\mathcal{I}_{1}=\frac{1}{2} \zeta(2 \sigma, \alpha) T-\frac{2(2 \pi)^{\sigma-\frac{1}{2}}\left(1-2^{\sigma-\frac{3}{2}}\right)}{(2 \sigma-1)(3-2 \sigma)} T^{\frac{3}{2}-\sigma}+O\left(T^{1-\sigma} \log T\right) .
$$

Now

$$
\mathcal{I}_{2}=\sum_{0 \leq m, n \leq q(T)} \frac{e(\alpha(m-n))}{(m+\lambda)^{1-\sigma}(n+\lambda)^{1-\sigma}} \int_{T_{2}}^{T}\left(\frac{t}{2 \pi}\right)^{1-2 \sigma} \exp \left(i t \log \frac{n+\lambda}{m+\lambda}\right) d t
$$

with $T_{2}:=\max \left\{\frac{T}{2}, 2 \pi(m+\lambda)^{2}, 2 \pi(n+\lambda)^{2}\right\}$. For the diagonal terms we get

$$
\begin{gathered}
\frac{(2 \pi)^{2 \sigma-1}}{2-2 \sigma} \sum_{0 \leq n \leq q(T)}(n+\lambda)^{2 \sigma-2}\left(T^{2-2 \sigma}-\max \left\{\frac{T}{2}, 2 \pi(n+\lambda)^{2}\right\}^{2-2 \sigma}\right) \\
=\frac{(2 \pi)^{2 \sigma-1}}{2-2 \sigma}\left(T^{2-2 \sigma} \sum_{0 \leq n \leq q(T)}(n+\lambda)^{2 \sigma-2}-\left(\frac{T}{2}\right)^{2-2 \sigma} \sum_{0 \leq n \leq q\left(\frac{T}{2}\right)}(n+\lambda)^{2 \sigma-2}\right. \\
\left.-(2 \pi)^{2-2 \sigma} \sum_{q\left(\frac{T}{2}\right)+1 \leq n \leq q(T)}(n+\lambda)^{2-2 \sigma}+O\left(T^{1-\sigma}\right)\right) .
\end{gathered}
$$


For $\sigma=1 / 2$ this is equal to

$$
\frac{T}{4} \log \frac{T}{\pi}+\frac{T}{2}\left(c(\lambda)-\frac{1}{2}\right)+O\left(T^{1 / 2}\right)
$$

and for $1 / 2<\sigma<1$, to

$$
\begin{array}{ll}
\frac{(2 \pi)^{2 \sigma-1}\left(1-2^{2 \sigma-2}\right)}{2-2 \sigma} \zeta(2-2 \sigma, \lambda) T^{2-2 \sigma}+\frac{2(2 \pi)^{\sigma-\frac{1}{2}}\left(1-2^{\sigma-\frac{3}{2}}\right)}{(2 \sigma-1)(3-2 \sigma)} T^{\frac{3}{2}-\sigma} & \\
& +O\left(T^{1-\sigma}\right) .
\end{array}
$$

Lemma 3 yields for $m \neq n$

$$
\int_{T_{2}}^{T}\left(\frac{t}{2 \pi}\right)^{1-2 \sigma} \exp \left(i t \log \frac{n+\lambda}{m+\lambda}\right) d t \ll T^{1-2 \sigma}\left|\log \frac{n+\lambda}{m+\lambda}\right|^{-1} .
$$

Therefore, we find for the non-diagonal terms

$$
\begin{aligned}
& \sum_{\substack{0 \leq m, n \leq q(T) \\
m \neq n}} \frac{e(\alpha(m-n))}{(m+\lambda)^{1-\sigma}(n+\lambda)^{1-\sigma}} \int_{T_{2}}^{T}\left(\frac{t}{2 \pi}\right)^{1-2 \sigma} \exp \left(i t \log \frac{n+\lambda}{m+\lambda}\right) d t \\
& \ll T^{1-2 \sigma} \sum_{0 \leq m<n \leq q(T)}(m+\lambda)^{\sigma-1}(n+\lambda)^{\sigma-1}\left(\log \frac{n+\lambda}{m+\lambda}\right)^{-1} .
\end{aligned}
$$

By use of Lemma 2 this is $\ll T^{1-\sigma} \log T$. Thus,

$$
\mathcal{I}_{2}=\frac{T}{4} \log \frac{T}{\pi}+\frac{T}{2}\left(c(\lambda)-\frac{1}{2}\right)+O\left(T^{1 / 2} \log T\right), \quad \text { for } \sigma=1 / 2
$$

and

$$
\begin{aligned}
\mathcal{I}_{2}= & \frac{(2 \pi)^{2 \sigma-1}\left(1-2^{2 \sigma-2}\right)}{2-2 \sigma} \zeta(2-2 \sigma, \lambda) T^{2-2 \sigma}+\frac{2(2 \pi)^{\sigma-\frac{1}{2}}\left(1-2^{\sigma-\frac{3}{2}}\right)}{(2 \sigma-1)(3-2 \sigma)} T^{\frac{3}{2}-\sigma} \\
& +O\left(T^{1-\sigma} \log T\right), \quad \text { for } 1 / 2<\sigma<1 .
\end{aligned}
$$

Since the argument $g(\lambda, \alpha, t)$ of $\psi$ in $\mathcal{I}_{3}$ is bounded, we get easily $\mathcal{I}_{3} \ll T^{1-\sigma}$. Further, we have $\mathcal{I}_{4} \ll T^{\sigma-1}$. Now we consider

$$
\mathcal{I}_{5} \ll \sum_{0 \leq m, n \leq q(T)}(m+\alpha)^{-\sigma}(n+\lambda)^{\sigma-1}\left|\int_{T_{5}}^{T} t^{\frac{1}{2}-\sigma} e^{i f(t)} d t\right|
$$

with $T_{5}:=\max \left\{\frac{T}{2}, 2 \pi(m+\alpha)^{2}, 2 \pi(n+\lambda)^{2}\right\}$ and

$$
f(t):=t \log \frac{t}{2 \pi e(m+\alpha)(n+\lambda)} .
$$


Lemma 4 gives in combination with Lemma 3

$$
\int_{T_{5}}^{T} e^{i f(t)} d t \ll\left\{\begin{array}{c}
T^{\frac{1}{2}}, \\
\left(\log \frac{\max \left\{(m+\alpha)^{2},(n+\lambda)^{2}\right\}}{(m+\alpha)(n+\lambda)}\right)^{-1} .
\end{array}\right.
$$

The function $t^{\frac{1}{2}-\sigma}$ is decreasing and positive. Therefore, application of the second mean value theorem for the real and the imaginary part separately, leads with regard to (7) and $T \asymp T_{5}$ to

$$
\int_{T_{5}}^{T} t^{\frac{1}{2}-\sigma} e^{i f(t)} d t \ll T^{\frac{1}{2}-\sigma}\left|\int_{T_{5}}^{T} e^{i f(t)} d t\right| .
$$

This yields

$$
\begin{aligned}
\mathcal{I}_{5} \ll & T^{\frac{1}{2}-\sigma} \sum_{\substack{0 \leq m, n \leq q(T) \\
|m-n| \geq 2}}(m+\alpha)^{-\sigma}(n+\lambda)^{\sigma-1}\left(\log \frac{\max \left\{(m+\alpha)^{2},(n+\lambda)^{2}\right\}}{(m+\alpha)(n+\lambda)}\right)^{-1} \\
& +T^{1-\sigma} \sum_{\substack{0 \leq m, n \leq q(T) \\
|m-n| \leq 1}}(m+\alpha)^{-\sigma}(n+\lambda)^{\sigma-1} \\
\ll & T^{\frac{1}{2}-\sigma}\left(\sum_{0 \leq m<n \leq q(T)}(m+\alpha)^{-\sigma}(n+\lambda)^{\sigma-1}\left(\log \frac{n+\lambda}{m+\alpha}\right)^{-1}\right. \\
& \left.+\sum_{0 \leq n<m \leq q(T)}(m+\alpha)^{-\sigma}(n+\lambda)^{\sigma-1}\left(\log \frac{m+\alpha}{n+\lambda}\right)^{-1}\right) \\
& +T^{1-\sigma}\left(1+\sum_{1 \leq m \leq q(T)} m^{-1}\right) .
\end{aligned}
$$

Using Lemma 2, we obtain once more $\mathcal{I}_{5} \ll T^{1-\sigma} \log T$. Now

$$
\begin{aligned}
\mathcal{I}_{6} & \ll\left|\int_{\frac{T}{2}}^{T} t^{-\frac{\sigma}{2}} e^{-\pi i f(\lambda, \alpha, \sigma+i t)} \psi(g(\lambda, \alpha, t)) \sum_{0 \leq n \leq m(t)} \frac{e(\lambda n)}{(n+\alpha)^{\sigma+i t}} d t\right| \\
& \ll \sum_{0 \leq n \leq m(T)}(n+\alpha)^{-\sigma}\left|\int_{T_{6}}^{T} H(t) e^{i h(t)} d t\right|
\end{aligned}
$$

with $T_{6}:=\max \left\{\frac{T}{2}, 2 \pi(n+\alpha)^{2}\right\}$ and

$$
\begin{aligned}
H(t) & :=t^{-\frac{\sigma}{2}} \psi(g(\lambda, \alpha, t)), \\
h(t) & :=-t \log (n+\alpha)-\pi f(\lambda, \alpha, \sigma+i t) .
\end{aligned}
$$


Define $l:=q(t)-m(t)$, then $l$ is constant 0 or 1 . We divide $\left[T_{6}, T\right]$ into subintervals on which $l$ and $q:=q(t)$ are constant, namely

$$
l=\left\{\begin{array}{ll}
1 & , \quad t \in J_{1}(q):=\left[2 \pi q^{2}, 2 \pi(q+\alpha)^{2}\right) \cap\left[T_{6}, T\right] \\
0 & , \quad t \in J_{0}(q):=\left[2 \pi(q+\alpha)^{2}, 2 \pi(q+1)^{2}\right) \cap\left[T_{6}, T\right]
\end{array} .\right.
$$

Therefore

$$
\mathcal{I}_{6} \ll \sum_{0 \leq n \leq m(T)}(n+\alpha)^{-\sigma} \sum_{0 \leq l \leq 1} \sum_{n+1-l \leq q \leq q(T)}\left|\int_{J_{l}(q)} H(t) e^{i h(t)} d t\right| .
$$

If $q \leq n+2$, then

$$
\int_{J_{l}(q)} H(t) e^{i h(t)} d t \ll \int_{2 \pi q^{2}}^{2 \pi(q+1)^{2}} t^{-\frac{\sigma}{2}} d t \ll(q+1)^{1-\sigma} .
$$

Hence

$$
\begin{aligned}
& \sum_{0 \leq n \leq m(T)}(n+\alpha)^{-\sigma} \sum_{0 \leq l \leq 1} \sum_{n+1-l \leq q \leq n+2}\left|\int_{J_{l}(q)} H(t) e^{i h(t)} d t\right| \\
& \ll \sum_{0 \leq n \leq m(T)}(n+\alpha)^{1-2 \sigma} \ll T^{1-\sigma} .
\end{aligned}
$$

For $q \geq n+3$ we will use (4). Therefore, we calculate

$$
\begin{aligned}
h^{\prime}(t) & =\log \frac{\left(\frac{t}{2 \pi}\right)^{\frac{1}{2}}}{n+\alpha}-\frac{l+\{\lambda\}-\alpha}{2}\left(\frac{t}{2 \pi}\right)^{-\frac{1}{2}} \asymp \log \frac{q}{n+\alpha}, \\
h^{\prime \prime}(t) & =\frac{1}{2 t}+\frac{l+\{\lambda\}-\alpha}{8 \pi}\left(\frac{t}{2 \pi}\right)^{-\frac{3}{2}} \ll q^{-2}
\end{aligned}
$$

and

$$
\begin{aligned}
H(t) & \ll q^{-\sigma} \\
H^{\prime}(t) & =-\frac{\sigma}{2} t^{-\frac{\sigma}{2}-1} \psi(g(\lambda, \alpha, t))+t^{-\frac{\sigma}{2}} \psi^{\prime}(g(\lambda, \alpha, t)) \cdot \frac{1}{2 \pi}\left(\frac{t}{2 \pi}\right)^{-\frac{1}{2}} \ll q^{-\sigma-1} .
\end{aligned}
$$

Now (4) leads to

$$
\int_{J_{l}(q)} H(t) e^{i h(t)} d t \ll q^{-\sigma}\left(\log \frac{q}{n+\alpha}\right)^{-1} .
$$

Thus,

$$
\begin{aligned}
& \sum_{0 \leq n \leq m(T)}(n+\alpha)^{-\sigma} \sum_{0 \leq l \leq 1} \sum_{n+3 \leq q \leq q(T)}\left|\int_{J_{l}(q)} H(t) e^{i h(t)} d t\right| \\
& \ll \sum_{0 \leq n<q-2 \leq q(T)}(n+\alpha)^{-\sigma} q^{-\sigma}\left(\log \frac{q}{n+\alpha}\right)^{-1} \ll T^{1-\sigma} \log T
\end{aligned}
$$


with the help of Lemma 2. Summing up, we have $\mathcal{I}_{6} \ll T^{1-\sigma} \log T$. The integral $\mathcal{I}_{7}$ can be treated similar. We have

$$
\mathcal{I}_{7} \ll \sum_{0 \leq n \leq q(T)}(n+\lambda)^{\sigma-1}\left|\int_{T_{7}}^{T} K(t) e^{i k(t)} d t\right|
$$

with $T_{7}:=\max \left\{\frac{T}{2}, 2 \pi(n+\lambda)^{2}\right\}$ and

$$
\begin{aligned}
K(t) & :=t^{\frac{1}{2}-\frac{3 \sigma}{2}} \psi(g(\lambda, \alpha, t), \\
k(t) & :=-t \log \frac{t}{2 \pi e(n+\lambda)}-\pi f(\lambda, \alpha, \sigma+i t) .
\end{aligned}
$$

With the same arguments as above one gets after some calculations

$$
\begin{aligned}
\mathcal{I}_{7} & \ll \sum_{0 \leq n \leq q(T)}(n+\lambda)^{\sigma-1} \sum_{0 \leq l \leq 1} \sum_{n+1-l \leq q \leq n+2} q^{2-3 \sigma} \\
& +\sum_{0 \leq n \leq q(T)}(n+\lambda)^{\sigma-1} \sum_{0 \leq l \leq 1} \sum_{n+3 \leq q \leq q(T)} q^{1-3 \sigma} \log \left(\frac{q}{n+\lambda}\right)^{-1} \\
& \ll \sum_{0 \leq n \leq q(T}(n+\lambda)^{1-2 \sigma}+\sum_{0 \leq n<q-2 \leq q(T)}(n+\lambda)^{\sigma-1} q^{1-3 \sigma}\left(\log \frac{q}{n+\lambda}\right)^{-1} \\
& \ll T^{1-\sigma} \log T .
\end{aligned}
$$

Using the Cauchy-Schwarz inequality and (6) we have

$$
\mathcal{I}_{8} \ll\left(\int_{\frac{T}{2}}^{T} t^{\sigma-2} d t\right)^{\frac{1}{2}}\left(\int_{\frac{T}{2}}^{T}\left|\sum_{0 \leq n \leq m(t)} \frac{e(\lambda n)}{(n+\alpha)^{\sigma+i t}}\right|^{2} d t\right)^{\frac{1}{2}} \ll\left\{\begin{aligned}
T^{\frac{1}{4}} \log ^{\frac{1}{2}} T, & \text { for } \sigma=\frac{1}{2} \\
T^{\frac{\sigma}{2}}, & \text { for } \frac{1}{2}<\sigma<1 .
\end{aligned}\right.
$$

Further,

$$
\mathcal{I}_{9} \ll\left(\int_{\frac{T}{2}}^{T} t^{\sigma-2} d t\right)^{\frac{1}{2}}\left(\int_{\frac{T}{2}}^{T} t^{1-2 \sigma}\left|\sum_{0 \leq n \leq q(t)} \frac{e(-\alpha n)}{(n+\lambda)^{1-\sigma-i t}}\right|^{2} d t\right)^{\frac{1}{2}} \ll T^{\frac{1}{4}} \log ^{\frac{1}{2}} T .
$$

Finally, one easily gets $\mathcal{I}_{10} \ll 1$. Summing up, we obtain

$$
\int_{\frac{T}{2}}^{T}|L(\lambda, \alpha, 1 / 2+i t)|^{2} d t=\frac{T}{2} \log \frac{T}{\pi}+\frac{T}{2}(c(\alpha)+c(\lambda)-1)+O\left(T^{1 / 2} \log T\right)
$$

and for $1 / 2<\sigma<1$,

$$
\begin{aligned}
\int_{\frac{T}{2}}^{T}|L(\lambda, \alpha, \sigma+i t)|^{2} d t= & \frac{1}{2} \zeta(2 \sigma, \alpha) T+\frac{(2 \pi)^{2 \sigma-1}\left(1-2^{2 \sigma-2}\right)}{2-2 \sigma} \zeta(2-2 \sigma, \lambda) T^{2-2 \sigma} \\
& +O\left(T^{1-\sigma} \log T+T^{\frac{\sigma}{2}}\right) .
\end{aligned}
$$

Using the mean square formulas above with $2^{-k} T$ instead of $T$ and adding up over all non-negative integers $k$, the assertion of the theorem follows. 


\section{Applications}

In $[5]^{1}$ we defined for $0<\alpha \leq 1, \lambda \in \mathbb{R}, Q \in \mathbb{Z}$ the twist of the Lerch zeta-function $L(\lambda, \alpha, s)$ with a Dirichlet character $\chi \bmod q$ by

$$
L(\lambda, \alpha, s, \chi, Q)=\sum_{n=0}^{\infty} \frac{\chi(n+Q) e(\lambda n)}{(n+\alpha)^{s}} \quad(\sigma>1) .
$$

This generalizes Dirichlet $L$-functions $L(s, \chi):=\sum_{n=1}^{\infty} \frac{\chi(n)}{n^{s}}$. Those twists have an analytical continuation throughout the whole complex plane except for at most one simple pole at $s=1$, which follows immediately from the representation

$$
L(\lambda, \alpha, s, \chi, Q)=\frac{1}{q^{s}} \sum_{0 \leq k<q} \chi(k+Q) e(\lambda k) L\left(\lambda q, \frac{k+\alpha}{q}, s\right) .
$$

Summing up over all characters $\chi \bmod q$, we get

$$
\frac{1}{\varphi(q)} \sum_{\chi \bmod q}|L(\lambda, \alpha, s, \chi, Q)|^{2}=\frac{1}{q^{2 \sigma}} \sum_{\substack{0 \leq k<q \\(k+Q, q)=1}}\left|L\left(\lambda q, \frac{k+\alpha}{q}, s\right)\right|^{2} .
$$

Thus, applying the mean square formula, we obtain

Corollary 5 For $0<\lambda, \alpha \leq 1$ we have, as $T$ turns to infinity

$$
\begin{aligned}
& \frac{1}{\varphi(q)} \sum_{\chi \bmod q} \int_{1}^{T}|L(\lambda, \alpha, 1 / 2+i t, \chi, Q)|^{2} d t \\
& \quad=\frac{\varphi(q)}{q} T \log \frac{T}{2 \pi}+\frac{T}{q}\left(\varphi(q) c(\lambda q)+\sum_{\substack{0 \leq k<q \\
(k+Q, q)=1}} c\left(\frac{k+\alpha}{q}\right)-\varphi(q)\right)+O\left(T^{\frac{1}{2}} \log T\right)
\end{aligned}
$$

and for $\frac{1}{2}<\sigma<1$,

$$
\begin{aligned}
& \frac{1}{\varphi(q)} \sum_{\chi \bmod q} \int_{1}^{T}|L(\lambda, \alpha, \sigma+i t, \chi, Q)|^{2} d t \\
& \quad=\frac{1}{q^{2 \sigma}} \sum_{\substack{0 \leq k<q \\
(k+Q, q)=1}} \zeta\left(2 \sigma, \frac{k+\alpha}{q}\right) T+\frac{\varphi(q)}{q^{2 \sigma}} \frac{(2 \pi)^{2 \sigma-1}}{2-2 \sigma} \zeta(2-2 \sigma,\{\lambda q\}) T^{2-2 \sigma} \\
& \quad+O\left(T^{1-\sigma} \log T+T^{\frac{\sigma}{2}}\right) .
\end{aligned}
$$

\footnotetext{
${ }^{1}$ After the preparation of [5] it becomes known to the authors that Berndt [1] obtained partly similar, and in a joint paper with Schoenfeld [2] in some parts even more general results.
} 


\section{References}

[1] B.C. Berndt, Character analogues of the Poisson and Euler-Mac Laurin summation formulas with applications, J. Number Theory 7 (1975), 413-445

[2] B.C. Berndt, Schoenfeld, Periodic analogues of the Euler-Mac Laurin and Poisson summation formulas with applications to number theory, Acta Arithmetica 28 (1975), 23-68

[3] R. Garunkštis, A. LaurinčIKas, On the Lerch zeta-function, Lith. Math. J., 36(4) (1996), 337-346

[4] R. Garunkštis, A. LaurinČikas, J. Steuding, An approximate functional equation for the Lerch zeta-function, submitted for publication, preprint is available at http://www.mif.vu.lt/ttsk/bylos/ga/ga_a.html

[5] R. Garunkštis, J. Steuding, On twists of Lerch zeta-functions, submitted for publication, preprint is available at http://www.mif.vu.lt/ttsk/bylos/ga/ga_a.html

[6] A. HuRwitz, Einige Eigenschaften der Dirichletschen Funktionen $F(s)=$ $\sum\left(\frac{D}{n}\right) \frac{1}{n^{s}}$, die bei der Bestimmung der Klassenzahlen binärer quadratischer Formen auftreten, Zeitschrift f. Math. u. Physik 27 (1887), 86-101

[7] A. Ivić, The Riemann zeta-function, John Wiley \& Sons 1985

[8] D. KLusch, Asymptotic equalities for the Lipschitz-Lerch zeta-function, Arch. Math. 49 (1987), 38-43

[9] A. LAURinčIKAS, On the mean square of the Lerch zeta function, LMD mokslo darbai 3 (1999), 62-67

[10] M. LERCH, Note sur la fonction $\mathcal{K}(z, x, s)=\sum_{k=0}^{\infty} e^{2 k \pi i x}(z+k)^{-s}$, Acta Math. 11 (1887), 19-24

[11] H.L. Montgomery, R.C.Vaughan, Hilbert's inequality, J. London Math. Soc. 8 (1974), 73-82

[12] V.V. RAne, On the mean square value of Dirichlet L-series, J. London Math. Soc. (2), 21 (1980), 203-215 